\title{
Application of the simulated annealing algorithm to minimize the makespan on the unrelated parallel machine scheduling problem with setup times
}

\author{
GabrielaAmaral $^{1}$, Lino Costa $^{1}$, Ana Maria A. C. Rocha ${ }^{1}$, Leonilde Varela ${ }^{1}$ and Ana \\ Madureira $^{2}$ \\ ${ }^{1}$ Algoritmi Research Centre, University of Minho, 4800-058 Guimarães, Portugal \\ ${ }^{2}$ Interdisciplinary Studies Research Center (ISRC), ISEP/IPP, Portugal \\ id5731@alunos.uminho.pt, $\{$ lac, arocha, leonilde $\}$ dps. uminho.pt, amd@ \\ isep.ipp.pt
}

\begin{abstract}
In this paper, the unrelated parallel machine scheduling problem considering machine-dependent and job sequence-dependent setup times is addressed. This problem involves the scheduling of $n$ jobs on $m$ unrelated machines with setup times in order to minimize the makespan. The Simulated Annealing algorithm is used to solve four sets of small scheduling problems, from the literature, on two unrelated machines: the first one has six jobs, the second has seven jobs and the third and fourth has eight and nine jobs, respectively. The results seem promising when compared with other methods referred in literature.
\end{abstract}

Keywords: Scheduling Problem, Setup Times, Simulated Annealing.

\section{Introduction}

The production scheduling when optimizing configurations, either directly or indirectly, has been an important area for the various types of industries, including plastic, textile, and chemical, as well as some service areas [1-9]. In addition, it has been an area of great importance in research, expressed through a vast set of scientific publications [2-5]. Aydilek et al. [10] address a scheduling problem to minimize order delays, in which the configuration times independently of the order processing times, with the application of algorithms of self- adaptive Differential Evolution and a hybrid and simulated insertion algorithm. The scheduling problem with different approaches to change or setup times, with the objective of reducing complexity in order to minimize the makespan or the total production time, with the application of an enhanced version of the ant colony optimization algorithm was studied in $[11,12]$.

The problem of parallel machine programming has been a growing research area since the early work [13]. In Arnaout et al. [14], it was used an Ant Colony optimization algorithm for minimizing the schedule's makespan on unrelated parallel machine with sequence-dependent setup times. For the same problem and objective but considering the setup times, Rabadi et al. [1] introduced a new metaheuristic (MetaRaPS) and its 
performance was evaluated by comparing its solutions with an existing heuristic for the same problem. The results show that MetaRaPS found all optimal solutions for small problems and outperformed the solutions obtained by the existing heuristics for larger problems. The unrelated parallel machine scheduling problem (PMSP) when minimizing the makespan was addressed by Woo et al. [15] where a mixed integer linear programming (MILP) model to find the optimal solution was developed. They proposed a novel rule based on the Genetic Algorithm (GA) with a chromosome representing job assigning sequence to one of the machines and the schedule of jobs completion time rule based on dispatching heuristics during the decoding process of the chromosome. Abreu's work [16] described a hybrid GA for solving the unrelated parallel machine scheduling problem with sequence dependent setup times. A case study on the granite industry is presented and the proposed approach outperformed three traditional dispatch rules presented in the current literature. Gedik et al. [17] studied the non-preemptive unrelated parallel machine scheduling problem with job sequence and machine dependent setup times with the objective of minimizing the makespan. This study provided a novel constraint programming (CP) model with two customized branching strategies that uses CP's global constraints, interval decision variables, and domain filtering algorithms. In terms of average solution quality, the computational results indicated that the CP model slightly outperforms all of the state-of-art algorithms in solving small problem instances and was able to prove the optimality of 283 currently best-known solutions. It is also effective in finding good quality feasible solutions for the larger problem instances. Fanjul-Peyro et al. [18] studied the same problem with the same objective function but they modelled the problem by means of two integer linear programming problems. One is based on a model previously proposed in the literature and the other is based on the resemblance to strip packing problems. Since the models were unable to solve medium-sized instances to optimality, they proposed three Metaheuristics strategies for each of these two models. The results show that the Metaheuristics significantly outperform the mathematical models.

Recently, a multi-objective approach about parallel machines was proposed as a new problem, the resource constrained unrelated parallel machine green manufacturing scheduling problem with the criteria of minimizing the makespan and the total carbon emission [19]. To solve this problem, a collaborative multi-objective fruit fly optimization algorithm was proposed. The results showed that the multi-objective algorithm was able to obtain more and better non-dominated solutions than other algorithms.

Considering the previous literature review, most of the research addressed the scheduling problem with different objectives and algorithms application in different contexts and industrial environments.

The purpose of this work is motivated by the Rabadi et al. [1] work about the truss manufacturing industry where manufacturing roof trusses requires different setup times depending on the manufacturing sequence and the type of manufacturing apparatus used. Based on data for small instances [1] we propose a Simulated Annealing (SA) algorithm for solving the unrelated parallel machine scheduling problem considering the setup times with the objective of minimizing the makespan, $C_{\text {max }}$. In order to assess the performance of this algorithm when solving this kind of problems, a comparison with the solutions obtained by the metaheuristic MetaRaPs in [1] will be made. 
Thus, in this study, we intend to evaluate the behaviour of the SA algorithm when solving four types of case studies of unrelated parallel machine scheduling problems with setup times. The first is a collection of 15 instances for two machines and six jobs, the second, 15 instances for two machines and seven jobs, 15 instances for two machines and eight jobs, 15 instances for two machines and nine jobs.

This paper is organized as follows. In Section. 2, the problem formulation is briefly described, by explaining the main constraints behind the variables and parameters of the problem. In Section 3, the results of the numerical experiments are presented and Section 4 contains the conclusions of the present study and some ideas for future work.

\section{Problem Formulation}

This article addresses the unrelated parallel machine scheduling problem considering the scheduling of $n$ jobs that are available at time zero on $m$ unrelated machines $\left(R_{m}\right)$. The objective function is the $C_{\max }$, taking into account machine-dependent and sequence dependent setup time $S_{i j k}$. When the job's processing times depend on the machines to which they are assigned, and it does not exist any relationship between machine speeds, the machines are unrelated. The setup time $S_{i j k}$ depends on the jobs sequence and the machine because each machine has its own matrix of setup times. The type of problem discussed in this article is classified in the literature as $R_{m}\left|S_{i j k}\right| C_{m a ́ x}$ [20]. The basic identical PMSP $P_{m} \| C_{m a ́ x}$ is NP-hard even when $m=2$ [21-23]. We considered that $R_{m}\left|S_{i j k}\right| C_{m a ́ x}$ is also NP-hard since it is a generalization of the former problem [20].

Below we describe the problem used the following assumptions and notations:

- $\quad m$ is the number of parallel machines of the set M;

- $n$ denotes the number of jobs to be scheduled of the set $\mathrm{N}$;

- Each machine can only process one job at a time without interruption;

- For the initial instant that it is at time zero all jobs are available. No restrictions of precedence are imposed among jobs;

- In each machine $k$, each job $i$ has a processing time $p_{i k}$;

- In each machine $k$, for processing job $j$ just after job $i$, there is a setup time $s_{i j k}$. The setup time is different for each machine.

- The objective is to minimize the makespan $C_{\max }$. The term span is used to define the completion time of a machine while the term makespan is used for the maximum span in the solution of the problem.

The nomenclature presented to describe the machines and jobs can be flexible, between $i, j, k$, as well as the variables describing the sets of machines and jobs ( $\mathrm{M}$ and $\mathrm{N})$. In this work, we have four case studies involving 2 machines $(m=2)$ and $n=6$, $n=7, n=8$ and $n=9$ jobs. 


\section{$3 \quad$ Results and discussion}

In this section, we analyse the behaviour of the SA algorithm when solving unrelated parallel machine scheduling problems considering the setup times and the objective function to be minimized is $C_{\max }$. The instances were taken from [1] and are only considered some small problems and balanced times data belonging to two case studies, each one composed by a collection of 15 instances: the first case for two machines and six jobs, the second for two machines and seven jobs, the third for two machines and eight jobs and the fourth for two machines and nine jobs. The data used is available at Scheduling Research Virtual Centre Homepage [24, 25].

The SA algorithm has undergone some changes to the original algorithm, available at Academic Source Codes and Tutorials [24], developed by S. Mostapha Kalami Heris for parallel machine scheduling problem. The original code of the algorithm was adapted to consider the setup time of the first job, the maximum number of iterations was fixed to 100 iterations within the specific parameters of SA to gain at run time. For each case study and for each instance 20 runs were done, since SA is a stochastic algorithm. The run time, $C_{\text {max }}$ and Work Order are outputs of the algorithm SA. For each case study and for each run associated to each instance, two types of graphs can be generated: the work order and the $C_{\max }$ along the iterations. The case studies were run in the MatLab ${ }^{\circledR}$ R2017 using a PC i7-4600U CPU @ 2.10GHz 2.70GHz.

Table 1 shows the average values of $C_{\max }$ (Avg. $C_{\max }$ ) and run time (Avg Time) obtained by SA when solving each of the 15 instances among the 20 runs.

Table 1. Average solutions of $C_{\max }$ and run time

\begin{tabular}{c|cc|cc|cc|cc}
\hline \multirow{2}{*}{ Instance } & \multicolumn{2}{|c|}{$m=2, n=6$} & \multicolumn{2}{c|}{$m=2, n=7$} & $m=2, n=8$ & \multicolumn{2}{c}{$m=2, n=9$} \\
\cline { 2 - 9 } & Avg. $C_{\max }$ & Avg Time & \multicolumn{2}{c}{ Avg. } & Avg Time & Avg. $C_{\text {mín }}$ Avg Time & Avg. $C_{\max }$ & Avg Time \\
\hline 1 & 390.00 & 390.30 & 484.00 & 486.25 & 494.00 & 498.90 & 627.00 & 645.00 \\
2 & 410.00 & 410.10 & 527.00 & 531.00 & 523.00 & 523.00 & 603.00 & 609.60 \\
3 & 410.00 & 411.60 & 498.00 & 499.70 & 508.00 & 510.30 & 558.00 & 565.70 \\
4 & 380.00 & 380.70 & 467.00 & 470.90 & 539.00 & 546.95 & 618.00 & 622.60 \\
5 & 399.00 & 399.80 & 495.00 & 497.00 & 509.00 & 512.55 & 571.00 & 588.00 \\
6 & 397.00 & 397.00 & 493.00 & 493.55 & 502.00 & 504.75 & 589.00 & 596.20 \\
7 & 394.00 & 394.20 & 470.00 & 470.80 & 522.00 & 523.80 & 611.00 & 619.40 \\
8 & 379.00 & 379.00 & 516.00 & 522.05 & 534.00 & 536.45 & 624.00 & 625.80 \\
9 & 407.00 & 407.00 & 496.00 & 496.00 & 518.00 & 525.15 & 594.00 & 613.80 \\
10 & 394.00 & 395.50 & 490.00 & 493.90 & 517.00 & 526.80 & 616.00 & 614.10 \\
11 & 388.00 & 388.00 & 489.00 & 489.00 & 511.00 & 525.20 & 579.00 & 584.00 \\
12 & 396.00 & 401.30 & 483.00 & 485.80 & 509.00 & 512.10 & 588.00 & 598.30 \\
13 & 384.00 & 385.10 & 493.00 & 493.50 & 505.00 & 510.25 & 599.00 & 611.50 \\
14 & 369.00 & 370.20 & 488.00 & 504.40 & 563.00 & 565.40 & 612.00 & 616.30 \\
15 & 424.00 & 424.60 & 476.00 & 480.95 & 507.00 & 507.00 & 594.00 & 608.00 \\
\hline
\end{tabular}


In order to evaluate the performance of SA when solving this set of unrelated parallel machine problems with sequence-dependent setup times, a comparison with another metaheuristic will be done. Thus, Table 2 shows the solutions obtained by MetaRaPs in [1] and the best values of $C_{\max }\left(\right.$ Best $C_{\max }$ ) obtained by SA when solving each of the 15 instances, among the 20 runs.

Table 2. Best solutions of $C_{\max }$ obtained by SA and MetaRaPs

\begin{tabular}{c|cc|cc|cc|cc}
\hline \multirow{2}{*}{ Instance } & \multicolumn{2}{|c|}{$m=2, n=6$} & \multicolumn{2}{c|}{$m=2, n=7$} & \multicolumn{2}{c|}{$m=2, n=8$} & \multicolumn{2}{c}{$m=2, n=9$} \\
\cline { 2 - 8 } & MetaRaPs & Best $C_{\text {max }}$ & MetaRaPs & Best $C_{\text {max }}$ & MetaRaPs & Best $C_{\text {max }}$ & MetaRaPs & Best $C_{\text {max }}$ \\
\hline 1 & 390.00 & 390.00 & 484.00 & 484.00 & 494.00 & 494.00 & 627.00 & 627.00 \\
2 & 410.00 & 410.00 & 527.00 & 527.00 & 523.00 & 523.00 & 603.00 & 603.00 \\
3 & 410.00 & 410.00 & 498.00 & 498.00 & 508.00 & 508.00 & 558.00 & 558.00 \\
4 & 380.00 & 380.00 & 467.00 & 467.00 & 539.00 & 539.00 & 618.00 & 618.00 \\
5 & 399.00 & 399.00 & 495.00 & 495.00 & 509.00 & 509.00 & 571.00 & 571.00 \\
6 & 397.00 & 397.00 & 493.00 & 493.00 & 502.00 & 502.00 & 589.00 & 589.00 \\
7 & 394.00 & 394.00 & 470.00 & 470.00 & 522.00 & 522.00 & 611.00 & 611.00 \\
8 & 379.00 & 379.00 & 516.00 & 516.00 & 534.00 & 534.00 & 624.00 & 624.00 \\
9 & 407.00 & 407.00 & 496.00 & 496.00 & 518.00 & 518.00 & 594.00 & 594.00 \\
10 & 394.00 & 394.00 & 490.00 & 490.00 & 517.00 & 517.00 & 616.00 & 612.00 \\
11 & 388.00 & 388.00 & 489.00 & 489.00 & 511.00 & 511.00 & 579.00 & 579.00 \\
12 & 396.00 & 396.00 & 483.00 & 483.00 & 509.00 & 509.00 & 588.00 & 588.00 \\
13 & 384.00 & 384.00 & 493.00 & 493.00 & 505.00 & 505.00 & 599.00 & 599.00 \\
14 & 369.00 & 369.00 & 488.00 & 488.00 & 563.00 & 563.00 & 612.00 & 610.00 \\
15 & 424.00 & 424.00 & 476.00 & 476.00 & 507.00 & 507.00 & 594.00 & 594.00 \\
\hline
\end{tabular}

Analysing Table 2, it can be concluded that the solutions between the two metaheuristics MetaRaPS and SA are very similar. However, it was verified that for the case of two machines and nine works, for the instances 10 and 14, the solutions obtained by SA are better than the ones from MetaRaPS, reaching the optimal known solution.

We noticed that for instance 10 with $m=2, n=9$, the SA obtained the best solution in 17 of the 20 runs, giving nine different working orders for the same global best solution of $C_{\max }=612$. Figure 1 shows the work sequence that was assigned to the case study for $m=2, n=9$, instance 10 , for the different global solutions. In the figure it is possible to visualize which and how many jobs were allocated to each machine and which machine finishes the execution of the planned tasks, the best achieved $C_{\text {max }}$. The works positioned in the lower part of the figure refer to the machine 1 , and those positioned in the upper part, to the machine 2 . The empty spaces between the beginning and the end of each job, refer to the setup time allocated between each job. 

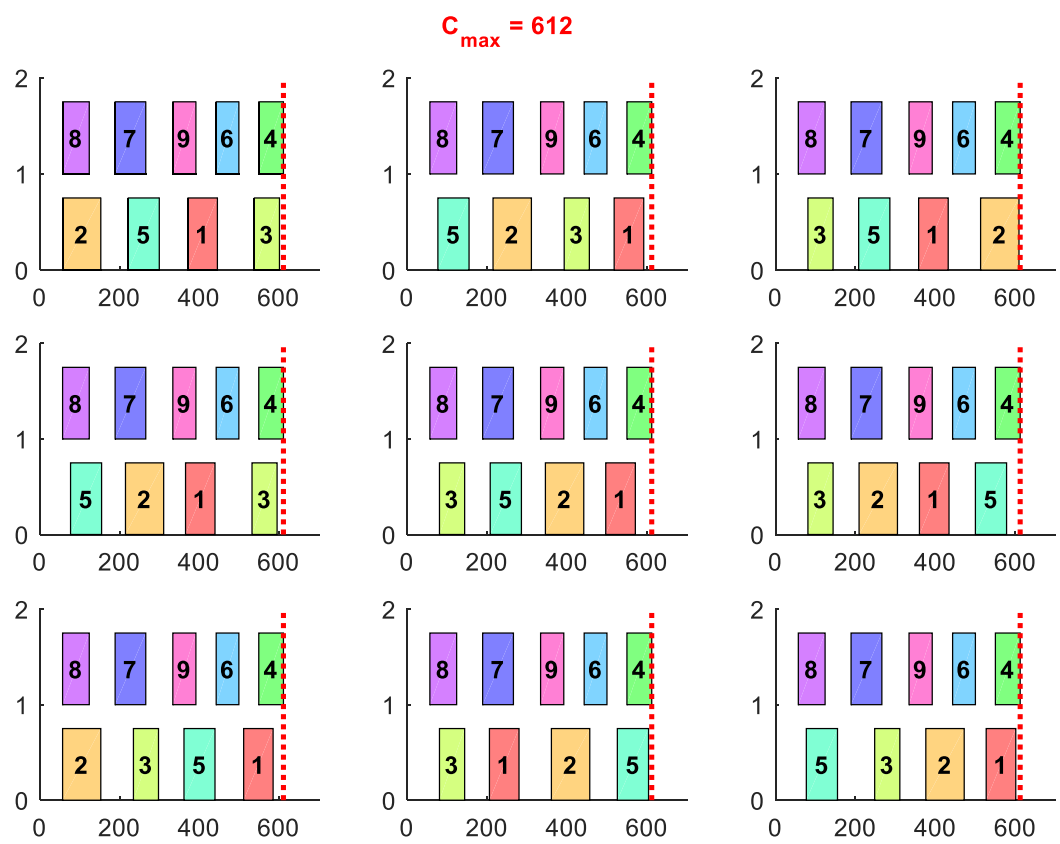

Fig. 1. Work Order of instance 10 for $m=2$ and $n=9$

For instance 14 of the case of two machines and nine jobs, the best solution was obtained in 10 out of the 20 runs, giving in all of them the same working order for a best value of $C_{\max }=610$, as shown in Figure 2 .

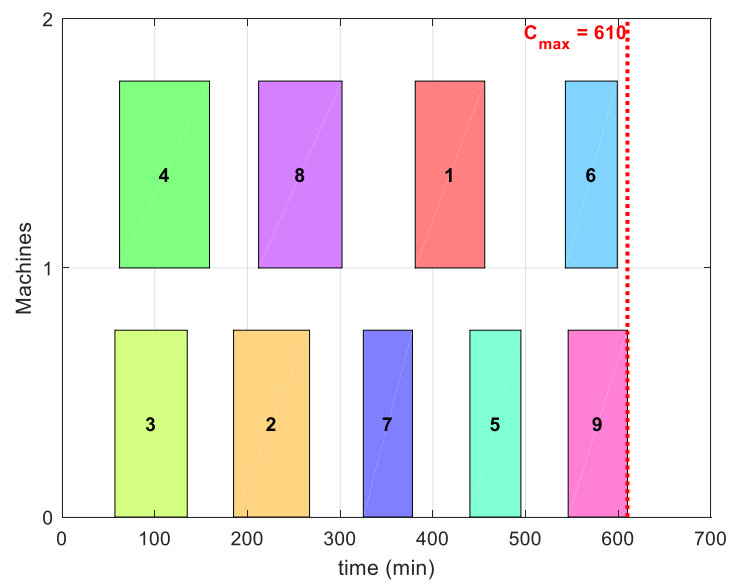

Fig. 2. Work Order of instance 14 for $m=2$ and $n=9$ 
Figure 3 shows the value of $C_{\max }$ along the iterations for instance 14 with $m=2$ and $n=9$. In the first 20 iterations, a noticeable decrease in the $C_{\max }$ value is observed. For the remaining iterations, the $C_{\max }$ value remains constant for about 60 iterations and a last decrease is observed near iteration 90 .

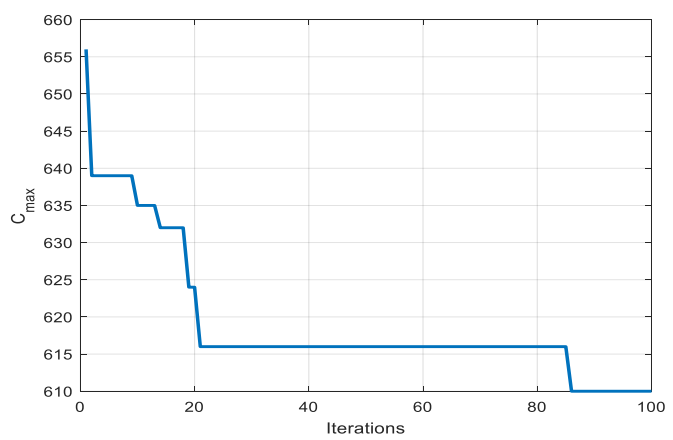

Fig. 3. $C_{\max }$ along the iterations of instance 14 for $m=2$ and $n=9$

Figure 4 shows the average and best values of $C_{\max }$ of the 15 instances, for the case study of two machines $(m=2)$ and six jobs $(n=6)$. For almost all the instances the average of $C_{\max }$ values is close to the best values, except for instance 12 . Figure 5 plots the average versus best of $C_{\max }$ over the 15 instances, for the case study of $m=2$ and $n=7$. It presents a large difference between the average and best values for some instances. For the case study of $m=2$ and $n=8$, when showing the differences between the average and best values of $C_{\max }$, for the 15 instances, Figure 6 reveals higher differences for almost all instances. For two machines $(m=2)$ and nine jobs $(n=9)$, in Figure 7, it is observed that the difference from average and best values of $C_{\max }$ has increased for the 15 instances when compared to the other cases.

Consistently, Figure 4 to Figure 7 show that when the number of jobs increases, the differences between the average and best values of $C_{\max }$ also increase. This behaviour can be due to the search space that becomes also larger with the number of jobs. In spite of the growth of the search space, it should be noted that the maximum number of iterations of SA was maintained for all problems. In these figures, we also observe a considerable variability of the average and best values of $C_{\max }$ that can be caused by the big difference between the data assigned to each instance.

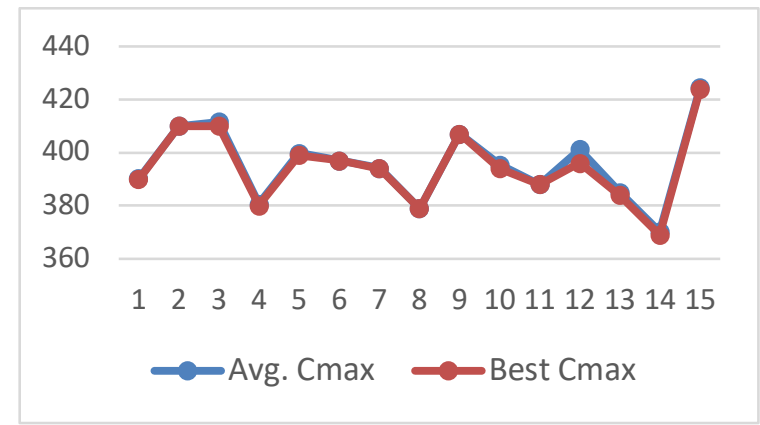

Fig. 4. Average $C_{\text {max }}$ vs Best $C_{\text {max }}$ for $m=2, n=6$ 


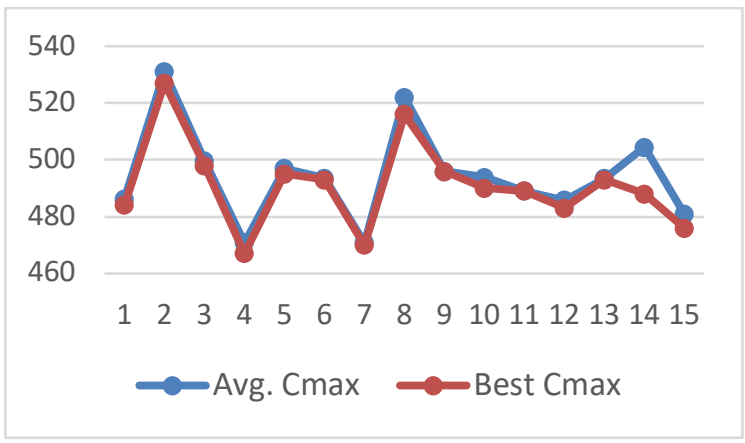

Fig. 5. Average $C_{\text {max }}$ vs Best $C_{\text {max }}$ for $m=2, n=7$

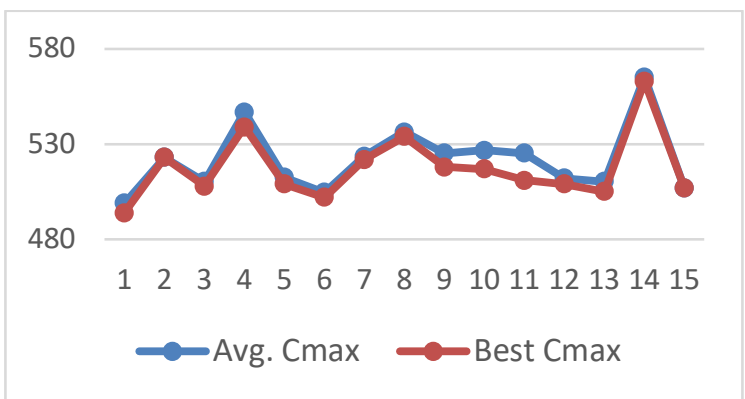

Fig. 6. Average $C_{\text {max }}$ vs Best $C_{\text {max }}$ for $m=2, n=8$

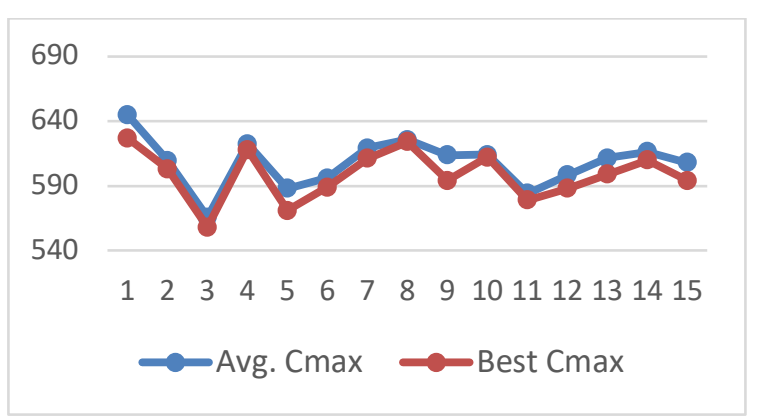

Fig. 7. Average $C_{\max }$ vs Best $C_{\max }$ for $m=2, n=9$

\section{Conclusions and future work}

The area of scheduling problem to optimize setup time has been and remains important for the industries and research. In this work, we intended to study the unrelated parallel machine scheduling problem with the objective of minimizing the $C_{\max }$, considering machine-dependent and job sequence-dependent setup times. This type of problem is classified as $R_{m}\left|S_{i j k}\right| C_{\max }$ and the basic identical PMSP $P_{m} \| C_{\max }$ is NP-hard even when $m=2$ [21]. 
Radabi et al. [1] studied the same problem with the same objective, and introduced a new metaheuristic, the MetaRaPs. In this paper, we used the simulated annealing optimization algorithm for minimizing the $C_{\max }$, of four types of case studies: two machines with a number of jobs varying between six and nine. For each case study, 15 instances were considered and solved by SA algorithm. The results obtained were compared with those obtained by MetaRaPs [1] to evaluate the behaviour of the SA algorithm. The solutions obtained by the two metaheuristics MetaRaPS and SA were very similar. However, it was verified that for the case of two machines and nine works, in two instances, the solutions obtained by SA were better than the ones from MetaRaPS, reaching the optimal known solution. We remark that, for some instances, SA was able to find different working orders for the same global best solution. The performance of SA was also studied when the number of jobs increases. Consistently, it was observed that the differences between the average and best values of $C_{\max }$ increase with the number of jobs as the search space becomes larger.

In near future we aim to improve the SA and tune its parameters in order to reduce the time execution. In addition, it is intended to analyse the behaviour the SA through the introduction of more case studies by varying the number of machines and jobs.

\section{Acknowledgement}

This project is funded by COMPETE: POCI-01-0145-FEDER-007043 and FCT Fundação para a Ciência e Tecnologia within the project scope: UID/CEC/00319/2013.

\section{References}

1. Rabadi, G., Moraga, R. J., \& Al-Salem, A. (2006). Heuristics for the unrelated parallel machine scheduling problem with setup times. Journal of Intelligent Manufacturing, 17(1), 85-97.

2. Varela, M. L. R., Aparício, J. N., \& Silva, S. C. (2003). A Web-based application for manufacturing scheduling. In IASTED International Conference on Intelligent Systems and Control (pp. 400-405).

3. Reddy, M. S., Ratnam, C., Agrawal, R., Varela, M. L. R., Sharma, I., \& Manupati, V. K. (2017). Investigation of reconfiguration effect on makespan with social network method for flexible job shop scheduling problem. Computers \& Industrial Engineering, 110, 231-241.

4. Ghadiri Nejad, M., Güden, H., Vizvári, B., \& Vatankhah Barenji, R. (2018). A mathematical model and simulated annealing algorithm for solving the cyclic scheduling problem of a flexible robotic cell. Advances in Mechanical Engineering, 10(1).

5. Hinder, O., \& Mason, A. J. (2017). A novel integer programing formulation for scheduling with family setup times on a single machine to minimize maximum lateness. European Journal of Operational Research, 262(2), 411-423.

6. Nikabadi, M., \& Naderi, R. (2016). A hybrid algorithm for unrelated parallel machines scheduling. Int. Journal of Industrial Engineering Computations, 7(4), 681-702.

7. Rewers P., Trojanowska J., Diakun J., Rocha A., Reis L.P., A study of priority rules for a levelled production plan; In: Hamrol A., Ciszak O., Legutko S., Jurczyk M. (eds) 
Advances in Manufacturing. Lecture Notes in Mechanical Engineering. Springer, 2018, pp.111-120

8. Trojanowska J., Kolinski A., Galusik D., Varela M.L.R., Machado J., A methodology of improvement of manufacturing productivity through increasing operational efficiency of the production process. In: Hamrol A., Ciszak O., Legutko S., Jurczyk M. (eds) Advances in Manufacturing. Lecture Notes in Mechanical Engineering. Springer, 2018, pp.23-32

9. Bülbül, K., \& Şen, H. (2017). An exact extended formulation for the unrelated parallel machine total weighted completion time problem. Journal of Scheduling, 20(4), 373389.

10. Aydilek, A., Aydilek, H., \& Allahverdi, A. (2017). Minimising maximum tardiness in assembly flowshops with setup times. Int. Journal of Production Research, 1-25.

11. Zhang, S., \& Wong, T. N. (2016). Studying the impact of sequence-dependent set-up times in integrated process planning and scheduling with E-ACO heuristic. International Journal of Production Research, 54(16), 4815-4838.

12. Xu, L., Wang, Q., \& Huang, S. (2015). Dynamic order acceptance and scheduling problem with sequence-dependent setup time. International Journal of Production Research, 53(19), 5797-5808.

13. McNaughton, R. (1959). Scheduling with deadlines and loss functions. Management Science, 6(1), 1-12.

14. Arnaout, J. P., Musa, R., \& Rabadi, G. (2014). A two-stage Ant Colony optimization algorithm to minimize the makespan on unrelated parallel machines - part II: enhancements and experimentations. Journal of Intelligent Manufacturing, 25(1), 43-53.

15. Woo, Y. B., Jung, S., \& Kim, B. S. (2017). A rule-based genetic algorithm with an improvement heuristic for unrelated parallel machine scheduling problem with timedependent deterioration and multiple rate-modifying activities. Computers \& Industrial Engineering, 109, 179-190.

16. Abreu, L. R., \& Prata, B. A. (2018). A Hybrid Genetic Algorithm for solving the Unrelated Parallel Machine Scheduling problem with Sequence Dependent Setup Times. IEEE Latin America Transactions, 16(6), 1715-1722.

17. Gedik, R., Kalathia, D., Egilmez, G., \& Kirac, E. (2018). A constraint programming approach for solving unrelated parallel machine scheduling problem. Computers \& Industrial Engineering.

18. Fanjul-Peyro, L., Perea, F., \& Ruiz, R. (2017). Models and matheuristics for the unrelated parallel machine scheduling problem with additional resources. European Journal of Operational Research, 260(2), 482-493.

19. Zheng, X. L., \& Wang, L. (2018). A collaborative multiobjective fruit fly optimization algorithm for the resource constrained unrelated parallel machine green scheduling problem. IEEE Trans. on Systems, Man, and Cybernetics: Systems, 48(5), 790-800.

20. Pinedo, M. (2008). Scheduling- Theory, Algoritms, and Systems, 3rd edition, Prentice Hall,New Jersey.

21. Karp, R. M. (1972). Reducibility among combinatorial problems. In Complexity of computer computations (pp. 85-103). Springer, Boston, MA.

22. Garey, M. R., \& Johnson, D. S. (1979). Computers and intractability: a guide to NPcompleteness.

23. Rabadi, G. (Ed.). (2016). Heuristics, Metaheuristics and Approximate Methods in Planning and Scheduling (Vol. 236). Springer.

24. Academic Source Codes and Tutorials, www.yarpiz.com, last accessed 2018/09/29.

25. Scheduling Research Virtual Center Homepage,www.SchedulingResearch.com. 